\section{ADRIANA ASSINI. LA GIOIA DEL COLORE, IL PIACERE}

DELLA STORIA

ADRIANA ASSINI. THE JOY OF THE COLOUR, THE PLEASURE OF THE STORY

Letizia Casella Universidad de Génova

\section{Riassunto:}

Abstract:

Adriana Assunti è nata e vive a Roma. È Adriana Assunti was born in Rome and she traduttrice, acquerellista e scrittrice. Tramite is still living there. She is a translator, write i suoi quadri e i suoi libri vuole raccontare and watercolourist. Through her paintings delle storie. Il viaggio rappresenta una and books, she wants to tell some stories. caratteristica molto importante nel lavoro di Another important characteristic of the Adriana Assunti. Lucia Pizzarelli la definisce work or Adriana Assunti is represented by un'artista colta, raffinata e delicata. Alcune discovering the world. Lucia Pizzarelli claims delle opere importanti dell'autrice sono I that she is an educated and sensitive artist. delle opere importanti dellaut ice sono I that she is an educated and sensitive artist. Gilles che amava Jeanne, Nella foresta di Some of her most significant books are IGilles
Soignes, Lo scettro di seta, Le evangeliste di che amava Jeanne, Nella foresta di Soignes, Bruges. Lo scettro di seta, Le evangeliste di Bruges.

\section{Parole chiave:}

\section{KEY WORD:}

Translator, watercolourist, writer, discovering Traduttrice, acquerellista, scrittrice, viaggio, the world, educated, sensitive. colta, raffinata.

\section{L'artista}

Adriana Assini è nata, vive e lavora a Roma, dove ha conseguito il Diplôme d'Etudes Françaises dell'Università di Scienze Sociali di Grenoble. Traduttrice, acquerellista e scrittrice, ha al suo attivo numerose mostre in Italia e all'estero. Ha già pubblicato nove romanzi storici ed un racconto breve, ottenendo importanti riconoscimenti.

«La creatività a volte ha bisogno del colore, a volte del pensiero» e Adriana Assini affascina e cattura con entrambe le sue espressioni artistiche. Personalmente ho conosciuto prima i suoi quadri e poi i suoi libri. I suoi acquerelli trasmettono la gioia del colore: le tonalità brillanti dei suoi azzurri, i verdi, i rossi; impossibile non immergersi nel piacere di ammirare gli infiniti particolari che impreziosiscono le immagini. Colpiscono gli occhi delle sue figure femminili: quegli sguardi fermi, diretti, profondi, di personaggi che scrutano chi li guarda, trasmettono la consapevolezza di chi ha origini lontane e sembrano affermare la propria, vivissima presenza. Sono le figure maschili ad avere espressioni incerte, sguardi sfuggenti, rappresentando personaggi più deboli. Caratteristiche di genere che si ritrovano pari pari nella sua produzione letteraria.

Come hanno notato in molti, prima di me, i suoi quadri sono storie e le sue storie sono quadri, con pennellate precise che ne delineano i protagonisti. Adriana Assin ha il dono di possedere entrambe le capacità espressive in modo spiccato, grazie alle quali, generosamente, regala viaggi bellissimi alla fantasia di coloro che la conoscono. Sia con la pittura che con la scrittura riesce a catturare immediatamente chi si avvicina alle sue opere: i suoi acquerelli deliziano gli occhi, i suoi testi provocano un piacere sottile, grazie al quale ci si immerge in atmosfere lontane e suscitano la curiosità di voler conoscere meglio $i$ fatti che descrive. In alcune interviste l'autrice stessa spiega la combinazione delle sue espressioni artistiche:

Nel mio caso, parole e immagini hanno obiettivi comuni: raccontare storie, comunicare emozioni. Le contaminazioni tra le due forme di espressione artistica non sono frutto di un ragionamento a monte: abituata a intingere il pennello ni colori, mi viene naturale rappresentare le scene dei miei romanzi com nei coloni, "rasseguirsi di quadri. Cosi cone, quando dipingo, i miei acquerelli diventano "racconti visivi". Tuttavia, a fare la differenza fra cio che scrivo e cio che dipingo è il diverso uso dell'immaginazione: nei miei romanzi, a carattere storico, narro vicende realmente accadute, lasciando intervenire liberamente la fantasia soltanto laddove le "carte" tacciono, mentre nei miei acquerelli l'immaginazione è sovrana e prende spunto da miti, favole, archetipi.

Lamore per i viaggi, essendo nata sotto il segno del Sagittario, è un segno marcato dell'autrice, per la quale viaggiare è scoprire; imparare; ampliare gli orizzonti spostando sempre un po' più in là le "colonne d'Ercole"; confrontarsi con il nuovo, $\mathrm{l}^{\prime}$ insolito o il diverso, anche a soli cento $\mathrm{km}$ da casa. [... ] Ma viaggiare non 
è soltanto mettersi in macchina o prendere un aereo. La mente è forse il maggiore mezzo di trasporto a nostra disposizione e ci conduce dove vogliamo, nel tempo e nello spazio. Grazie ai miei romanzi, ho attraversato a cavallo le Fiandre, quando erano ancora ricche di mulini ad acqua e a vento; ho "assistito" ai fuochi d'artificio sparati da Caster (t) nelle sue avventurose battute di caccia alle tigri.

La passione per la Storia muove la scrittrice a raccontare vicende realmente accadute, che arricchisce con la fantasia quando i documenti scarseggiano e, come lei stessa dice: «unisco al piacere di guardare indietro, quello di fare un'indagine nella storia», perché «il romanziere si infila nei vuoti che lascia la storia».

Adriana Assini è una ricercatrice attenta, oltre che coltissima, capace di ricreare le atmosfere di altri tempi con garbo, rendendo partecipe il lettore fin dalle prime righe, coinvolgendolo, con grazia, nella vicenda

La forma espressiva con l'utilizzo delle frasi brevi, rende scorrevoli i suoi testi; il linguaggio, sempre adeguato alle diverse epoche narrate ed ai diversi ambienti sociali, denota un meticoloso studio delle abitudini e consuetudini verbali dei tempi andati, che la scrittrice riesce puntualmente a ricostruire in modo gradevole e colorito: pennellate, appunto, di tinte vivaci che non stancano mai.

All'attrazione iniziale verso i personaggi prescelti, aggiungo l'esigenza di restituirli al lettore come persone vive e vicine, al di là dei secoli che separano le loro vicende dalle nostre. Il mio è dunque un lavoro di ricerca storica ma anche di indagine psicologica, che mi porta a "dialogare" con i protagonisti dei miei romanzi, cercando di ricostruirne la personalità non soltanto attraverso l'interpretazione degli episodi, frasi, atteggiamenti che ci tramandano le cronache, ma anche colmando con la fantasia gli spazi vuoti. Accade perciò che io li osservi mentre si vestono, mangiano, passeggiano. E che li ascolti mentre si lamentano o gioiscono. Succede anche che io mi arroghi il diritto di leggerne i pensieri, raccoglierne le frustrazioni, indovinarne le speranze, per poter dare loro corpo, anima e voce, farne uomini e donne in "carne e ossa", con le loro luci e le loro zone d'ombra. È quindi un rapporto intenso quello che mi lega ai miei personaggi, che perdura poi nel tempo, ben oltre la vita standard del romanzo: stesura, pubblicazione, promozione.

Si cala interamente in altre epoche, analizzando animo umano, mentalità corrente, usi e costumi di periodi storici in determinate aree geografiche, per rendere al meglio l'ambientazione dei fatti narrati. Leggendo i suoi romanzi sembra davvero di camminare in quei luoghi, udendo voci, espressioni, proverbi e di vederne i colori e di gustarne i sapori. Adriana Assini non si limita a descrivere, entra nei personaggi e ne fa sentire le pulsazioni, le emozioni, i sospiri. La scrittrice compie indagini meticolose, su eventi e personaggi, perché
[...] la stesura di questo lungo racconto del mondo non è mai definitiva, né completamente obiettiva, essendo a volte limitata da carenze nelle fonti; in altre, influenzata dalla "faziosità" degli storici. In alcuni casi, una rilettura critica rigorosa di taluni eventi, una insperata scoperta archeologica o qualche fortunato ritrovamento di importanti documenti negli archivi e nelle biblioteche, sono consolidate da secoli. Se, dunque, il "cantiere" resta aperto, la Storia, inevitabilmente, si riscrive.

Stefano Valentini traccia una breve ma significativo profilo della sua abilità descrittiva:

Assieme a Laura Mancinelli, autrice "specializzata" in vicende medievali, e a grande Sebastiano Vassalli, la romana Adriana Assini rappresenta oggi uno dei migliori esempi nazionali di "scrittore storico", ovvero un narratore che per le sue capacità letterarie potrebbe dedicarsi a qualsiasi tipo di romanzo ma che, per predilezione personale, si cimenta volentieri con ambientazioni e personaggi ricavati direttamente dalla storia. Le sue opere, lo sa bene chi ne ha letta almeno una, non hanno nulla di didascalico o rievocativo, ma traggono semplicemente spunto da un luogo o da una situazione d'altri tempi per sviluppare trame coinvolgenti o del tutto originali, dove ad essere messa in primo piano è la definizione psicologica dei personaggi assunti a protagonisti. [...] La scrittura è pressoché perfetta del tutto (e sottolineiamo 'del tutto' fin nei minimi dettagli) ripulita dalle sue piccole incertezze che spessissimo si riscontrano negli autori non consacrati sue piccole Adriana Assini, vorremmo auspicare "non ancora consacrati"-dallatio -ma noi per Adriana As in che conta e dalla cosiddetta grande editoria. E perfetti sono l'uso della lingua ricca e accurata, elegantissima e priva di qualsiasi cacofonia, l'utilizzo impeccabile della punteggiatura (che per molti autori spesso è un azzardo), l'equilibrio esemplare della struttura narrativa quanto a tempi, scene, descrizioni.

Le attente analisi psicologiche condotte dall'autrice romana fanno sì che Semiramide, Giulia Tofana, Giovanna di Castiglia, Gilles de Rais o altri dei suoi personaggi riscritti, ci vengano incontro in una nuova veste, una dimensione molto più umana, diversa da quella a cui siamo abituati dai testi ufficiali; che ci facciano riflettere sugli avvenimenti che li coinvolsero e per i quali furono, spesso a torto e sommariamente, etichettati, «I miei personaggi sono tutti un po' controversi ed io ricerco una verità che gli è stata negata».

Adriana Assini predilige le figure femminili, i suoi testi trattano di «donne forti, disposte a pagare anche prezzialtissimi pur dinon piegare la testa, donne che combattono malgrado le sconfitte, che ci insegnano prima di tutto il valore dell'autostima, cioè a credere in se stesse anche quando si hanno tutti contro».

Come ben precisa Mercedes Gonzáles de Sande, traduttrice in spagnolo di Adriana Assini:

In tutte le sue opere, tanto letterarie come artistiche, confluiscono entrambe le passioni, poiché i suoi dipinti mostrano spesso personaggi letterari o storici, 
come scene relazionate con la scrittura, mentre nei suoi testi il lato cromatico emerge frequentemente illuminando le sue dettagliate descrizioni. [...] La parola e l'immagine, pertanto, per la nostra scrittrice, sono strettamente legate da un obiettivo comune, che è quello di comunicare emozioni e raccontare storie. Per questo motivo, come lei stessa afferma, abituata a coniugare entrambi i mezzi, scene delle sue novelle, accompagnate sempre da minuziosi dettagli, si presentano come una successione di quadri che il lettore può quasi contemplare, come se si trovasse davanti alla realtà stessa, mentre che, a sua volta, i suoi acquerelli, che contengono sempre una grande carica espressiva, rappresentano storie o "relazioni visuali", come lei stessa definisce. [...] Nelle opere di Assini possiamo ancora constatare le chiare impronte di una tradizione letteraria anteriore, dove la letteratura era il riflesso della verità, poiché scaturiva dall'interiorità umana, e il valore morale dell'opera contava più delle mere esibizioni formali. In definitiva la nostra scrittrice opta per una scrittura, ma anche per una pittura, che vanno oltre le apparenze per trovare significati e valori essenziali provenienti dall'animo umano. Per tale motivo, indaga nella psicologia dei suoi personaggi, tanto in quelli esistiti rer , pubblico, mostrandoci le loro inquietudini, le loro emozioni, le loro passioni piu nascoste, facendoci partecipi emotivamente nelle moltitudini di vite, quasi come se fossimo
presenti, fino al punto, certo, di poter giungere a identificarci con alcuni di essi».

\section{Così la definisce Lucia Pizzarelli:}

Colta e raffinata, artista sensibile e delicata, Assini vanta un curriculum di tutto rispetto: i suoi romanzi, come le sue mostre, in Italia e all'estero, suscitano consensi di pubblico e di critica. Nella scrittura e nella pittura, la medesima dimensione fiabesca in una lettura controcorrente della realtà evidenzia una costante tensione metaforica l'aspirazione a colmare la distanza tra il bene e il male, tra la morte, tra verità e menzogna, tra universo maschile e femminile.

Concludo questa breve presentazione, con le parole dell'autrice stessa, che riassumono una sua caratteristica: «C'è un legame molto stretto tra quello che scrivo e ciò che dipingo anche se nel secondo caso non scelgo mai soggetti storici. Eppure le due attività si incontrano, una frase, una parola diventano coma una pennellata che restituisce forme e colori di un'epoca. Ogni scenario che racconto è come se lo dipingessi».

\section{Le opere letterarie}

\subsection{Gilles, che amava Jeanne}

Il romanzo è ambientato nel XV secolo, epoca in cui un personaggio femminile di grande fascino e personalità come Giovanna d'Arco dominò sia la scena politica che quella religiosa. Di lei si innamorò perdutamente Gilles de Laval, sire di Rais, uomo raffinato, ricco, colto e potente. La condanna e la morte della pulzella d'Orléans sconvolsero terribilmente Gilles, che accusò Dio di avergli tolto la donna amata, e si vendicò contro il destino crudele seviziando e trucidando un enorme numero di ragazzini, i cui pianti disperati gli ricordavano le grida di Giovanna al rogo. Arrestato e giustiziato, invocherà l'amata fino alla fine della sua vita.

Si tratta sicuramente di un romanzo di difficile stesura che Adriana Assini ha saputo abilmente descrivere. Luciano Pirrotta, lo ha presentato magistralmente:

Può avvenire che fuori dai circuiti editoriali delle case che contano fioriscano talvolta piccoli gioielli letterari. Può accadere che, nonostante l'insufficiente distribuzione pressole maggiori librerie, taligemme solitarie riescano ugualmente distruinere gli estimatori Può infine succedere che senza la "supplica" a ll' pupplica" dellautore, "ce dell'edlo sed cosiddett "critci", il hibro, finanche acquistato e non hicevuto in omaggio sotto l'auspicio di una benevola recensione, attir lattenzione di chi dovrebbe solo segnalare al lettore l'uscita delle poche opere degne di nota nel mare magnum della carta stampata. Da questo fortunato concorso di circostanze scaturiscono le rapide notazioni che seguono. Il romanzo di Adriana Assini, dedicato alle fin troppo sfruttate figure di Gilles de Rais e Giovanna d'Arco, scava nelle pieghe vive della psicologia dei due condottieri, restituendoli problematici e vitali così come forse vissero e morirono. Proprio perché si tratta di personaggi storici mitizzati - la santa pastorella e il depravato infanticida - su cui sono corsi fium d'inchiostro, maggiormente difficile si presentava il compito di tentarne ulterio d proci che non tocassion approce chembra essere riuscita appieno, grazie alla preveribili mondo apparentemente molto distante da noi. Il sinistro signore di Rais e l'ispirata pulzella lorenese vengono restituiti alla sensibilità dei moderni fruitori attraverso pochi tratti essenziali, al pari dei luoghi e delle circostanze entro cui si muovono. Dalla irresistibile ascesa al repentino declino, alla fine precoce, la coppia guerriera si illumina vicendevolmente lungo un percorso costellato di afflati di santità e passioni non corrisposte, slanci generosi e vizi innominabili. $E^{\prime}$ stato merito dell'autrice trovare nel magma di una materia già di per sé complessa, lo spazio per lumeggiare con veloci cenni alcuni attori minori del dramma: gli ecclesiastici infidi, $i$ valletti conniventi, il pavido sovrano francese, l'alchimista Prelati, metà iniziato metà impostore. Il libro si legge con piacere, grazie allo stile "nobile" iniziato metá impostice "nobile" ma senza sbavature, il ritho serrato, mentre si coglie, fra le righe, lamore per le sfumature e la piena padronanza dell'argomen soltanto la "pulzella d"Orléans" ci appare più vicina nel suo estremo sacrificio, ma lo stesso "Sire di Rais" - nella triste attualità delle odierne cronache sulla pedofilia - acquista, pur deformata, una qualche sembianza di dolorosa umanità».

\subsection{Nella foresta di Soignes}

Il romanzo è ambientato nel 1482, quando, nel monastero agostiniano di RougeCloître (Roodekloster) presso Bruxelles, dove la sua infermità nervosa lo aveva condotto a chiedere ospitalità, muore Hugo van der Goes, uno dei grandi maestri della pittura fiamminga quattrocentesca. In quel luogo aveva trovato amicizia nel giovane novizio Sander Brückner, la cui vocazione monastica viene messa a rischio dalla clandestina 
frequentazione di Jean Deleuze, l'estroso brigante che percorre a cavallo i sentieri della vicina foresta di Soignes.

Avvenimenti dolorosi ed oscuri presagi spingono il novizio ad abbandonare il monastero per fuggire con il "re della foresta" inseguendo un sogno di libertà ma l'avventura lo condurrà a circostanze tragiche. Il brigante proseguirà il viaggio, intrecciando la propria sorte con quella di una folla di comprimari, dal saggio nano Gaston al capomastro Linard, umile e magistrale restauratore di cattedrali, alla cui arte finirà con l'associarsi.

Stefano Sacconi così commenta il testo:

Adriana Assini fa del racconto uno specchio finemente cesellato delle inquietudini e dei fermenti di un'epoca. La nuova vena pittorica, innanzi tutto, che dalle Fiandre e deifering e dalla vicina Olanda permea lEuropa fra le Alpi e il Mare delNord, dando avvio a un Rinascimento nordico per più versi intrecciato con quello mediterraneo che già da mezzo secolo fiorisce nell'Italia dei principati. E poi l'aspra lotta per il possesso delle menti e dei cuori, che già si sta avviando contro la religione istituzionale che con i suoi istituti ne ha disciplinato per secoli i moti e le aspirazioni; e che di lì a poco, cambiando protagonisti, esploderà in guerra aperta sotto la sferza impietosa di Lutero: i cui colpi non risparmieranno, di là dalla corruzione de poteri ecclesiastici, la stessa, alta visione laica di un Erasmo. Infine, il germinare negli uomini di un gusto nuovo della libertà, per la quale può già esservi qualche giovane animoso che osi sfuggire alle rassicuranti certezze della regola mettendo rischio, per rispondere alla sirena eslege di un affascinante avventuriero, londo a rischio, per risporiero, la stessa vita. L' autrice ci trasporta in quell atmosfera dicience ma lo fa con la consueta levita. I suoi personaggi, piu che essere scavati psicologicamente, appaiono come maschere eteree, figure multicolori tratte da un quadro tardogotico o ritagliate da un arazzo Gobelin. Essi ci parlano della loro epoca non attraverso un racconto estrinseco, ma in prima persona, con la lingua che presta loro la sottile erudizione della scrittrice (e acquerellista) romana. L'atmosfera rarefatta ed estraniata che ne nasce, invece di frapporsi fra noi e quei protagonisti d'una vicenda lontana nel tempo e ambientata nel lontano Brabante, c'inserisce in essa come testimoni incantati d'una scena in cui il giovane Sander sospeso fra due mondi, Jean il brigante ammaliatore, il sapiente e inquieto padre Claus, Gaston il saltimbanco, Yves l'alchimista e l'arista-muratore Linard sono protag, Gastid'un policromo teatro d'ombre. Una scena tanto irreale quanto coinvolgente an un policromo tetro donche per noi sospesi fra due secoli. in attesa forse che qualche Jeannot ci passi accanto sul suo bianco cavallo e ci persuada a fuggire dallo stazzo del conformismo (non precisamente monastico) che protegge la nostra prigionia, per affrontare in rischiosa libertà i verdeggianti e inesplorati sentieri della foresta di Soignes.

\subsection{Lo scettro di seta}

Il libro racconta le gesta della regina assira Semiramide, donna di straordinaria bellezza, collocata da Dante Alighieri a capo della schiera dei lussuriosi e citata dagli scrittori medievali quale esempio dello stesso vizio. La regina che ha ispirato Voltaire, Vivaldi, Rossini viene spesso ricordata soltanto per le sue imprese sessuali, ma già
Erodoto, e molto più tardi Christine de Pizan, scrissero del suo immenso valore e grande coraggio. Fondatrice di nuove città, tra cui Babilonia, fece costruire opere imponenti e fu la prima assira a far vedere il mare al suo popolo. Ritrovamenti archeologici e studi approfonditi, compiuti nel secolo scorso, le hanno reso giustizia portando alla luce documentazioni importanti del suo inestimabile operato.

Adriana Assini la racconta attraverso le emozioni, vissute in simbiosi con l'innata spinta all'agire razionale. Ne indaga il temperamento forte ed allo stesso tempo passionale: Semiramide è la donna che ama, ma che non si lascia annullare dall'amore. È capace di vivere e di godere della vita con passione e autodeterminazione, nella sua assoluta autenticità. Le sue gesta varcano i confini del tempo e dello spazio per offrirci una immagine femminile che in molte si vorrebbe imitare: riesce ad affrontare gli eventi senza permettere di esserne sopraffatta. Intelligente ed intraprendente, passionale ma non rinunciataria, che coltiva innanzitutto il rispetto e l'amore per se stessa.

\subsection{Il fuoco e LA CRETA. STORIE COMUNI NeLLA NAPOLI STRAORDiNARIA DEL 1799}

Adriana Assini ci offre uno scorcio della Napoli borbonica, nella quale non c'è spazio né per la speranza, né per la libertà individuale. L'amara solitudine accomuna diversi personaggi, sia popolani che aristocratici, tutti in balia del destino, che soffrono per la dolorosa incomunicabilità ed ai quali neppure l'amore offre spiragli di luce. La difficoltà dell'esistenza personale si riflette, o meglio riflette, quella sociale-storicopolitica. Come scrive Luciano Pirrotta:

Reazionari o rivoluzionari, borbonici o giacobini, tutti sono altrettante marionette d'un palcoscenico sinistro. Parassiti spagnoli e francesi si danno il cambio lungo la penisola italiana. Mai si cessera di allestire forche, di fucilare, di decapitare, di imprigionare in nome della libertà. Pare quasi che i popolani de Il fuoco e la creta (biereta gia presentan con matuati, proprio perche a pancia piena, delle fanfaluche ugualitarie e progressiste) che quei medesimi francesi, rapaci parolai di giustizia, saranno di lì a mezzo secolo i becchini della Repubblica Romana, alfieri della restaurazione pontificia; e che, qualche decennio dopo, la conclamata unificazione sotto la corona sabauda farà ben presto rimpiangere alle plebi meridionali - gravate da nuove gabelle vessatorie - l'antico 'malgoverno' spagnolo.

Ancora una volta l'autrice differisce nel delineare i personaggi ed attribuisce a quell femminili una maggiore determinazione. Gli uomini, seppur coraggiosi e generosi, non raggiungono mai il livello delle varie Jolanda, Lucia, Ruth. Nella riflessione di Werner «La vita scorre senza appartenerci e noi, modeste comparse, restiamo inermi e ininfluenti a guardare gli eventi» è contenuta tutta la loro fragilità.

Ancora una volta Adriana Assini riesce, con poche pennellate, a tratteggiare mirabilmente luoghi, situazioni e caratteri. Come sempre, la sua meticolosa ricerca, 
offre una ricostruzione linguistica eccellente, che rende il lettore partecipe degli avvenimenti.

\subsection{Il bacio del diavolo. Storia della contessa sanguinaria}

Adriana Assini ci conduce nell'Ungheria del XVI-XVII secolo, illustrandoci una società dove diritti e doveri dei singoli derivano dal ceto a cui, per nascita, si appartiene. In territori, faticosamente controllati dalla corona asburgica, insidiati dalle invasioni turche e dalle scorrerie dei nomadi delle steppe, vigono istituzioni, usi e costumi tipici dell'Alto Medioevo. Mentre in Occidente si vivono le stagioni dell'Umanesimo e del Rinascimento, con la fioritura delle arti e delle lettere, lì l'autorità arbitraria dei nobili sui sottoposti, è pressoché illimitata: uccidere un contadino perché non si è tolto il cappello innanzi ad un aristocratico, bastonare a morte un servo maldestro, abusare di donne ed ucciderle non costituisce, di fatto, effettivo reato. Le varie Chiese (cattolica, calvinista, luterana), sempre alleate del potere vigente, ratificano come volere di Dio la nascita dei derelitti.

In questo contesto, si colloca la figura di Erzsébet Báthory, la 'Signora di Csejthe' imputata di aver torturato e assassinato decine di fanciulle reclutate alle sue dipendenze in veste di serve o damigelle, di averne impiegato il sangue per abluzioni a scopo tonificante e cosmetico, di essersi spinta a pratiche di cannibalismo stregonesco. L'eroina del male finì i suoi giorni segregata in una stanza del castello di ?achtice, 'sepolta viva' per circa tre anni e mezzo, morì nel 1614, senza aver mai subito un processo ufficiale. Molti saggisti, narratori, drammaturghi, poeti, psicologi, medici, giuristi, si sono occupati di lei. La 'jena di Csejthe', la 'Contessa sanguinaria', la 'belva dei Carpazi', la 'vedova-vampiro'.

Lascio alle parole di Luciano Pirrotta, che ne ha curato la prefazione, il commento del testo:

L'autrice, che già in piccoli gioielli precedenti [...] si era calata in contesti cronologici molto precisi rievocandone accuratamente ambienti ed atmosfere, opera qui uno scavo profondo sulle figure di protagonisti, comprimari, comparse, della vicenda. Dire che si tratti di mera analisi psicologica sarebbe riduttivo, perché solo un puntuale studio bibliografico preventivo (che traspare pressoché ad ogni riga) può punce consentiri dischem rigorosi dell'attentillona Jó, Dorkó, Ficzkó riemergono restituiti a nuova vita mediante dettagli e sfumature elargiti con sapiente gradualità, risorga di pari passo il contesto sociale, geo-politico, culturale ove essi giocarono il loro ruolo rilevante o infimo. Attraverso la calibrata dosatura dei fitti dialoghi affiorano in una sorta di affresco progressivo non solo la piaggeria e la viltà dei subalterni, le incombenze quotidiane, le effimere contese locali ma, su uno scenario più vasto, intrighi e schermaglie che intessono la rete politico-diplomatica di buona parte della vecchia Europa centrale e, al contempo, la weltanschauung che pervade classi dominanti e sudditi, potenti e diseredati, alle prese gli uni con i problemi strategico-militari o dinastici, gli altri con le terribili difficoltà di sopravvivenza affrontate ogni giorno. Al centro della rappresentazione, lei, Erzsébet, all'esterno sprezzante, blasfema, decisa, spietata, eppure, nell'intimo, incerta, superstiziosa, lacerata, fragile creatura che assiste impotente alla procell che monta e finirà per travolgerla. .. Un discorso a sé, per quanto concerne la che me la delinezichenteconsigliera della contessa cui viene attribuita lescogitazione dei tormenti più efferati; qui l'autrice raggiunge davvero uno stato di grazia regalandoci un ritratto a tutto tondo della perfida suggeritrice, che farebbe bene a meditare perfino ch ha studiato la Báthory da un'angolazione accademica. Perché Anna (Annácska, Panni, Delbora o comunque la si voglia chiamare) è l'interfaccia di Erzsébet, il lascivo demone esteriorizzato delle sue pulsioni più inconfessabili continuamente bisbigliante la tentazione estrema: attingere l'assoluto tuffandosi nell'abisso senza fondo del male incondizionato. Adriana Assini ha colto in profondità questo aspetto complesso con una felicità di scrittura che attinge talvolta vertici di eccellenza. Vi si intravede in controluce la lezione di Freud e Jung Nietzsche ed Heidegger Sade e Bataille, quell'incontro con l'ombra' che ci attree e terrorizza perché la lotta piu e Bat quell' ardua è sempre il duello condotto al nostro interno, malgrado lo scontro continuo
con 'nemici' e insidie della realtà contingente.

\subsection{Le evangeliste di Bruges}

Un romanzo in cui l'autrice conferma le sue doti descrittive, grazie alle quali ci conduce in questo nuovo viaggio nella storia e ci affascina con concetti sospesi fra realtà e fantasia, fede e superstizione. Il contrasto sociale fa da sfondo al desiderio $\mathrm{d}$ conoscenza delle protagoniste. Le ribellioni e le lotte, seguite ai soprusi di un Contedespota, sono lo spunto che induce donne comuni ad incontrarsi clandestinamente nel tentativo di migliorare la realtà. La scrittrice ci fa prendere parte alla loro "Compagnia della Conocchia" ed al loro intento di riscrivere un "vangelo" rivolgendosi all'esoterico. La loro instancabile ricerca, per individuare sì i segreti del destino ma, soprattutto, per auspicare a cambiare la loro condizione umana: un futuro senza le tirannie di un Conte o di un padre padrone che imponga un matrimonio non voluto ad una figlia che ama un altro uomo.

Nel tratteggiare queste figure femminili, che non si arrendono e vogliono credere in un avvenire diverso, Adriana Assini dimostra padronanza psicologica, oltre che abilità e ricchezza linguistica.

Pacifico Topa così concludeva l'articolo di recensione del testo:

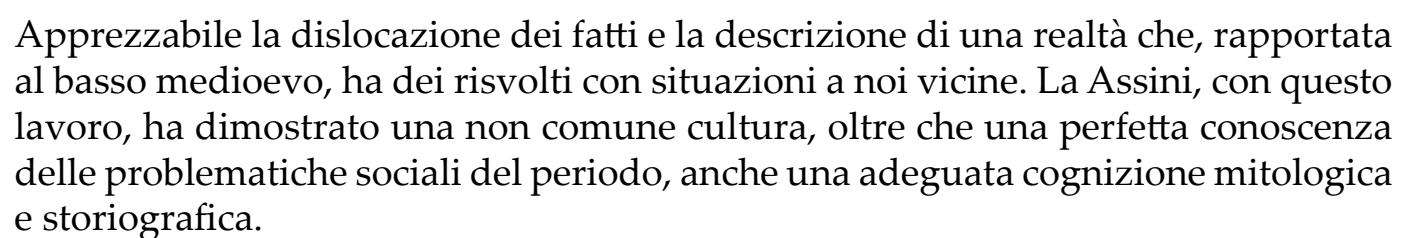
e storiografica. 


\subsection{Le rose di Cordova}

L'autrice ci porta nella Spagna del 1496, al momento della riconquista di Granada, dopo dieci anni di assedio, da parte dei Re Cattolici Isabella di Castiglia e Fernando d'Aragona, «abili nell'impugnare con medesima devozione sia le spade che i vangeli». La voce narrante del testo è Nura, figlia unica di Aziz el Sabio, primo ministro del Califfo, scelta da Giovanna di Castiglia, figlia dei conquistatori, come dama di servizio personale. A Nura, nobile e colta (conosce l'algebra e parla altre lingue) ma ora ridotta in schiavitù, è negato perfino il nome e viene ribattezzata Francisca, «un nome scelto a caso dal calendario cristiano». È l'unico personaggio inventato del romanzo, di cui si serve Adriana Assini per raccontare quasi mezzo secolo di Storia, quella ufficiale e quella volutamente ignorata. Attraverso la figura di quest'ancella la scrittrice ci rende partecipi di emozioni, angosce e sofferenze della sovrana che passò ai posteri come la Pazza e che non regnò neppure un giorno. Nel ricostruire la vita di Giovanna, l'autrice ci offre uno spaccato della storia europea davvero notevole, con la dovizia di particolari che ne caratterizzano lo stile, frutto, come sempre, della sua attenta e scrupolosa ricerca.

Adriana Assini propone un nuovo ritratto di Giovanna, attraverso una rilettura dei fatti documentati, spostando il punto d'osservazione, insinuando dubbi sulla follia, che fu motivo del suo mancato regno e, come noto, la costrinse relegata, torturata ed umiliata per quasi mezzo secolo. La sovrana assume un nuovo aspetto, di donna ribelle, ostinata, che neppure la lunga segregazione riesce a domare, circondata di persone assetate di potere che, facendola credere pazza, la escludono da ogni suo diritto.

L'idea di scrivere un libro su Giovanna di Castiglia viene così spiegata dall'autrice:

Nel corso di una ricerca in biblioteca, alle prese con l'Opus epistolarium di Pietro Martire d'Anghiera (cappellano di Isabella di Castiglia e storiografo ufficiale del "Nuovo Mondo"), mi soffermai sul resoconto di un drammatico episodio la cul "Nuovo Mondo"), mi sofferiso episodio la cui protagonista era Giovanna, la terzogenita dei Re Cattolici, passata alla storia come la "Pazza". Conoscevo le sue vicissitudini per sommi capi, ma dal racconto in questione ne ricavai la netta impressione che Juana, più che pazza, fosse stata invece una ribelle, destinata dunque a pagare a caro prezzo le sue "disobbedienze". La conferma mi venne poi a mano a mano che, nei mesi successivi, approfondii la sua storia per "riscriverla" sotto forma di romanzo e restituirle, anche se a posteriori, almeno un po' della dignità a cui aveva diritto Letizia.

Il romanzo è stato recentemente tradotto in spagnolo da Mercedes Gonzáles de Sande, appassionata studiosa dell'artista romana, che ha curato la prefazione del volume stampato in terra iberica, a cui attingo con piacere:

Tra "verità" storica e ipotesi personali, Adriana Assini, finalmente, riesce a rendere protagonista di quel periodo della storia di Spagna e d'Europa tanto controverso la regina Giovanna I di Spagna, conosciuta finora, più che per la sua rilevanza storica, per i suoi legami parentelari con alcune delle figure più emblematiche del nostro paese: figlia dei Re Cattolici, moglie di Filippo il Bello e madre di Carlo V.Il contesto storico che serve da scenario alle vicende personali di Giovanna I di Castiglia è quello di una Spagna ricca e potente, nel momento del suo massimo massimo splendore, mentre sta per convertirsi nell impero più vasto d'Europa. Una Spagna degli inizi del millecinquecento, piena di contraddizioni e di contrasti: da un lato, proiettata verso un futuro promettente grazie alla scoperta del Nuovo Mondo e per l'enorme peso politico che esercitava in Europa; dall'altro, bloccata da difficoltà economiche e sociali e dalla forte staticità di una società austera ed eccessivamente credente, controllata dall'Inquisizione, e anchilosata nelle idee religiose rispetto ad una Europa in piena ebollizione che s'incamminava verso l'Età Moderna. La scrittrice italiana ci presenta, con grande abilità e con un'ottima base documentale, un quadro perfettamente dettagliato di quel periodo, offrendo al lettore referenze storiche molto precise su date, luoghi, leggi, abitudini, avvenimenti [.. introducendoli nel testo come le marcate pennellate di uno dei suoi dipinti. [... Allo stesso modo, lungo la novella, si potranno scorgere dure critiche al razzismo, Allo ses. alla xenofobia, alla discrininazione sociale, razziale e di genere, alle ansie di potere capaci di radere al suolo tutto. In questo contesto tanto complesso e contraddittorio, tra politica e intrighi di corte, si muoveranno i protagonisti della novella, dei quali Adriana Assini traccerà un attentissimo ritratto psicologico, quasi come se li stesse spiando dal buco della serratura; un complesso intreccio da cui emergono la forza e il carattere dei principali personaggi, ma anche la loro fragilità e volubilità, così come le loro pene e angustie, le loro inquietudini e stati emozionali, mischiando, in tal modo, la storia ufficiale con la piccola storia quotidiana e privata di ciascun individuo, fatta di piccoli dettagli, di gesti, abitudini, sentimenti [...] In definitiva, la storia ufficiale resta in secondo piano, a favore della storia comune, creata da protagonisti, cercando di iffettere il lato più intimo e profondo di essi, quella essi, quella parte piu nascosta degli esseri umani che i documenti ufficiali non considerano, mostrando chiaramente le proprie debolezze e le proprie forze, in modo che il lettore possa giungere a comprendere il motivo delle loro azioni e trarre le proprie conclusioni, diversamente dalle cronache storiche, in cui tutto sembra inquestionabile. [...]Adriana Assini ci mostra nella sua novella una Giovanna di Castiglia ribelle e anticonformista, "pazza" per voler cambiare le regole imposte da una società con la quale non si sente identificata[...]. Una donna orgogliosa $\mathrm{d}$ essere com'era, che lottava per i suoi diritti, come nessuna donna aveva osato fare fino a quel momento, e che cercava di difendere i suoi valori ed i suoi principi al di sopra delle false apparenze .

\subsection{Un sorso di arsenico}

La storia è ambientata nel XVII secolo ed anche questa protagonista è una donna veramente esistita: si tratta della meretrice Giulia Tofana, «Venere plebea scolpita in marmo pario», capace di mettere a punto la formula del letale veleno che non lascia traccia sulle vittime. In un periodo in cui la condizione delle donne è molto difficile, Giulia attiva un notevole commercio delle proprie pozioni, liberando molte mogli da mariti violenti e opprimenti. Costretta a fuggire dalla natìa Palermo, dove regnano gli spagnoli e la peste, e dove rischia di essere scoperta, dopo una sosta a Napoli, giunge nella Roma in cui invece regnano lusso, potere e papa Urbano VIII. Un buon clima 
per riprendere i traffici interrotti ed annotare tra le proprie vittime circa 600 persone. Giulia è una donna tenace, che pur vivendo in ambienti malfamati, lotta per riscattarsi con i mezzi che possiede. Non rinnega nulla del suo operato e diventa la paladina delle donne oppresse. Il sottofondo della sua vita è l'amore: il barone Manfredi, atteso, inseguito, sfuggito, sognato, ritrovato, perduto. Per essere degna di quell'amore impara a leggere e scrivere, cresce, tenta di affinarsi e di cambiare vita. Ma su tutto vince l'amore per la libertà.

Adriana Assini offre un bell'affresco di quell'Italia, fondendo, con ironia, storia e invenzione, manonsi risparmianella descrizione di intrighi, aberrazioniecontraddizioni di quel tempo, che risultano sempre attuali. La figura di fra Nicodemo ben rappresenta il mondo di corruzioni, bassezze e condizionamenti da cui la rivoluzionaria Giulia, molto più coerente, vorrebbe fuggire.

Yvonne Aversa, scrive:

Adriana Assini, Arianna contemporanea, sa come condurci in un labirinto di passioni personali, storiche, sociali; $[. .$.$] ricostruisce fatti e personaggi con date$ e nomi presenti su tutti i libri di storia, aggiungendo sapientemente molto più di quanto si riferisce in essi. Voglio dire che mette in pratica, in tempi decisamente non sospetti di 'manzonismo', la teoria manzoniana -espressa nella fondamentale non sospetti di 'manzonismo', la teoria manzoniana -espressa nella fondamentale
"Lettera a Monsieur Chauvet"- sullo strettissimo, vitale, rapporto tra "storia e poesia". Riferendosi ai personaggi della Storia ufficiale, il padre del romanzo storico italiano sostenne -più o meno con queste parole- che '...i sentimenti che hanno accompagnato successi e insuccessi, i discorsi con i quali hanno cercato di far prevalere le loro passioni e le loro volontà...con i quali hanno manifestato, in una parola, la loro individualità, tutto ciò è passato sotto silenzio dalla storia, e tutto ciò forma il dominio della poesia. Il poeta svela ogni segreto dell'anima umana...il poeta può ...scorgerlo, afferrarlo ed esprimerlo.' A mio avviso è proprio questo... che poeta puo...scorgerlo, afferrarlo ed esprimerlo.' Amio avviso e proprio questo che affascina nel recente romanzo della Assini: la scrittrice rivela al lettore il 'dentro' dei personaggi: i loro pensieri, le emozioni, le percezioni, i sentimenti. [...] Certo, è vero che Giulia Tofana può essere vista come un personaggio 'minore' della storia del 1600. Ma è un personaggio che, a causa o grazie alla sua frequentazione di pozioni venefiche raggiunse una notorietà rilevante, tanto da lasciare il proprio cognome come indicativo d'uno dei più misteriosi e potenti veleni.

Mi riferisco specificamente a Mozart, del quale è noto che dichiarò che si accingeva alla composizione del notissimo Requiem 'per se stesso', perché convinto d'essere stato avvelenato con l'acqua Tofana per invidia e di esser condannato, dunque, a morire in breve tempo. E a Bulgakov, che chiaramente aveva notizia del personaggio e delle sue vicende tanto che se ne serví per accrescere l' atmosfera magica de "Il Maestro e Margherita".Difatti, nel capitolo XXIII del romanzo -"Il gran ballo da Satana"- tra gli invitati al sabba si descrive una donna «con una larga fascia verde al collo». Si tratta -spiega uno degli attendenti del diavolo- della signora Tofana, che "godeva di straordinaria popolarità tra le giovani, graziose napoletane, come pure tra le abitanti di Palermo, e in particolare fra quelle cui era venuto a noia il marito" [...] Adriana Assini ha realizzato un lavoro da certosino; non solo s'è documentata con accuratezza [...] ma ha anche ricostruito "verosimilmente" tutto un mondo corposo e molteplice ed è riuscita perfino a farlo parlare con un linguaggio che, legato fortemente ai tempi e agli spazi presenti nel romanzo, si muove su registri diversi e fortemente suggestivi, recuperando vocaboli e modi di dire d'altri tempi.

\subsection{Sogni di Vini}

Questo piccolo gioiello è un racconto breve, con il quale l'autrice, nel 2006, ha vinto il Premio Cesare Pavese - sezione inediti ed il Premio Verdicchio d'autore per inediti. La deliziosa prosa lo fa leggere d'un fiato. Il filo conduttore è il vino, dietro cui si nascondono profonde metafore. Non esistono indicazioni di tempi e luoghi, in tutto il testo viene pronunciato un solo nome, Bianca, attribuito per una sola notte all'unica figura femminile che vi compare.

Mentre la peste dilaga, in una taverna di un borgo sconosciuto, un araldo giunge per annunciare che il suo padrone, un principe solitario ed ignoto, offre ospitalità nel suo maniero, unico luogo al riparo dal contagio, a chiunque gli faccia dono di una giara di vino. Il vino però dovrà essere speciale, in grado di stupirlo « con un nuovo gusto per il suo palato» e che sia «in grado, per aroma, densità, colore e provenienza, di evocargli il fascino di terre lontane e corti sconosciute». Quattro uomini decidono di tentare l'avventura, poiché ciascuno possiede un vino di ottima qualità. Un mercante, un cavaliere, un frate ed un capitano: non si conoscono, ma si promettono aiuto reciproco per affrontare il viaggio e l'esperienza. Il principe li accoglie con un ricco banchetto e li mette a conoscenza del fatto che da secoli la sua famiglia produce e conserva vini eccellenti. Dopo aver loro offerto un assaggio delle sue prelibatezze, invita ognuno a parlare, prima di se stesso e poi del nettare che reca in dono. Al suo fianco ha la bellissima figlia, che gli ospiti decidono di chiamare Bianca, per concessione del padre poiché ella sostiene che «i nomi sono come il tempo, non contano. Bisogna lasciare che corrano via da noi, come l'acqua sotto i ponti». Gli ospiti raccontano, con sincerità, se stessi ed i loro vini, superando l'esame di accesso, e vengono invitati a trascorrere la notte nel castello. Dormiranno nella maestosa biblioteca, dove già alloggiano altre persone giunte prima di loro, con il medesimo intento.

«Chi sono questi signori che, al pari nostro, tra queste mura hanno trovato scampo da sicura morte e che in un'ora così tarda della notte ancora s'intrattengono tra le pagine di vecchi libri?» chiese allibito il frate. «Speziali, tessitori, maniscalchi e bottai, forgiatori, sarti scalpellini, tintori e scrivani:uomini diversi per età e mestiere,ma tutti ugualmente cultori del buon vino.» «Che fanno tutti insieme, in mezzo a tanti tomi?» fece timidamente il mercante, che in quella faccenda non ci vedeva chiaro. «Leggono 
e dunque, imparano. Ricordano e quindi, scrivono, "spiegò il Principe solitario «esercitano memoria e fantasia cercando le immagini e le parole da cui poi faranno scaturire nuove storie, capaci di saziare le mie orecchie e allietare il mio cuore in nome del dio vino».

Al risveglio i quattro si ritrovano all'addiaccio, increduli che castello e Principe siano potuti svanire nel nulla. Un contadino di passaggio spiega che quella è una terra d'incantesimi, abitata da spettri che, al calar della sera, «hanno poteri che noi non abbiamo e sono capaci di ricostruire con la mente il loro bel castello distrutto un giorno da un incendio. Al risveglio dall'incanto, alcuni dei loro ospiti impazziscono di nostalgia». I quattro ormai sono amici, uomini nuovi, che si separano con la promessa di non dimenticarsi, ma anzi di ritrovarsi nello stesso luogo a distanza di un anno, dopo la vendemmia, ognuno con il proprio vino migliore.

Ben vengano mecenati che aprano le loro porte a chiunque voglia mettersi in gioco e credere in un progetto comune: aiutarsi a vicenda nell'affrontare l'ignoto, offrire con sincerità ognuno il proprio vino, la vera essenza di sé, condividere il piacere di poter trascorrere del tempo insieme; cercare, acquisire e scambiarsi nuove conoscenze per crescere insieme, in compagnia della bellezza e della saggezza. Mai ci sarà banchetto più ricco. Poco importa che sia solo un'utopia, un sogno di una notte, l'importante è aver partecipato a quel sogno: la peste (etica e morale), che ci circonda, sarà comunque sconfitta! Adriana Assini ci porta in un luogo non luogo, tra personaggi senza nome, ad assaporare un nettare che allieta il cuore.

\subsection{Il mercante di zucchero}

L'autrice ci riporta nel secolo XVI, nella Sicilia dei vicerè spagnoli per narrarci un altro fatto storico realmente avvenuto, condito con un pizzico di fantasia. Il romanzo, diversamente dai precedenti, ha per protagonista un uomo, Gian Luca Squarcialupo, un mercante di zucchero e tonno appartenente ad una famiglia pisana trapiantata a Palermo, circondato da «un pugno di disperati, di poveri cristi che vivevano di stenti e non sarebbero mai arrivati ai quarant'anni». Non è affatto un santo, ma la sua indole ed i problemi economici, lo portano a guidare quella rivolta che finì nel sangue, l's settembre 1517. Con l'aiuto dell'amico fidato Cristoforo De Benedetto e l'appoggio della baronia locale, diventa capopopolo nella lotta contro le vessazioni del vicerè Hugo de Moncada, rappresentante di Carlo V.

Squarcialupo è un lottatore, «fa un uso spregiudicato delle armi eppure, nonostante questo, è un generoso e, dunque, vulnerabile. Dettaglio che potrebbe essergli fatale.» I suoi difetti lo rendono ancora più simpatico al lettore, è un passionale, un idealista. Il rapporto fraterno che lo lega a Cristoforo è un bell'esempio di amicizia incondizionata, quella che rassicura con uno sguardo, e dura fino alla morte.

L'unico, ma importante, personaggio femminile è quello di Francesca Campo, la donna di cui Gian Luca è da sempre innamorato: l'ha perduta in passato ed è intenzionato a riconquistarla con ogni mezzo. Adriana Assini ce la dipinge a tinte delicate, quasi sfumate ed in contrasto con quelle accese che usa per lo Squarcialupo, ma il sentimento che Francesca rappresenta è fortissimo ed è proprio quello che porta il protagonista ad agire, sognando un futuro insieme. In fondo il pensiero di lei è sempre presente nel testo, ed è ciò che stimola il mercante a crescere, a migliorarsi fino a diventare un eroe, ad appassionarsi ai problemi della sua gente ed a volerne difendere i diritti di libertà e giustizia.

Adriana Assini, con la meticolosità che la contraddistingue, ricostruisce nei minim dettagli la Palermo del tempo, con gli umori, gli intrighi piccoli e grandi, i tradimenti. Si cala nel passato per presentarci una realtà molto vicina a noi, ci descrive la setta dei Beati Paoli con una ricchezza di particolari perfino inquietanti, rendendoci spettatori di riti mafiosi mai svaniti.

Il ritmo della narrazione cresce via via fino all'epilogo tragico. Ma questa nostrana cronaca di una morte annunciata vale la pena di essere rivissuta passo passo. Non importa se poi «il popolo, ormai battuto ma ancora affamato, finì per rimettere la testa nel vecchio giogo dei potenti, laddove, d'altra parte, l'aveva sempre tenuta.». Lo spirito e le passioni che lo avevano animato non sparirono con le tracce di sangue cancellate in gran fretta il giorno successivo alla carneficina.

\section{Come scrive Marina Caracciolo:}

Ma se la rivolta del popolo palermitano si rivela destinata ad un amaro insuccesso, il fallimento e anche la morte nulla possono togliere agli ideali, al valore e alle nobili motivazioni di coloro che vi hanno partecipato combattendo. Adriana Assini - una scrittrice che, si può dire, dipinge anche quando scrive - sorretta come sempre dalla meticolosita e dalla ricchezza di documentazione che rimangono un imprescindibile punto di partenza in un romanzo che voglia definirsi storico, $\mathrm{ci}$ restituisce ancora una volta, e con consumata maestria, il variegato e brillante affresco di un' epoca, in questo romanzo politico e "corale" che sa avvincere il lettore dalla prima all'ultima pagina.

Lascio la pennellata finale a Paola Guarnieri:

Basato su un fatto realmente accaduto, Il mercante di zucchero prende spunto dalla storia, ma si affida all'invenzione narrativa per riempire i vuoti storiografici e dà vita ad un mosaico denso e suggestivo di eventi e personaggi che, grazie anche ad una scrittura coerente, intessuta di termini e proverbi tipici del tempo, ci trascina dentro uno scenario credibile, fatto di immagini, suoni, odori e colori.

\section{RIFERIMENTI BIBLIOGRAFICI}


Alighieri, D., La Divina Commedia - Inferno - canto V - vv. 52 e segg.

Assini, A., Gilles, che amava Jeanne, Tabula Fati, Chieti 1997.

----, Nella foresta di Soignes, Tabula Fati, Chieti 2001.

----, Lo scettro di seta, Tabula Fati, Chieti 2001.

----, Il fuoco e la creta. Storie comuni nella Napoli straordinaria del 1799, Spring, Caserta 2003.

----, Il bacio del diavolo. Storia della contessa sanguinaria, Spring, Caserta 2004.

----, Le evangeliste di Bruges, Tracce, Pescara 2004.

----, Le rose di Cordova, Scrittura \& Scritture, Napoli 2007.

----, Un sorso di arsenico, Scrittura \& Scritture, Napoli 2009.

----, Sogni diVini, Scrittura \& Scritture, Napoli 2011.

----, Las rosas de Cordoba, ArCiBel (su cortese concessione di Scrittura \& Scritture, Napoli), Sevilla 2011.

----, Il mercante di zucchero, Scrittura \& Scritture, Napoli 2011.

Aversa, Y., presentazione di Un sorso di arsenico su «Donneconoscenzastorica.it».

Caracciolo, M., recensione di Il mercante di zucchero su "Pomezia notizie", giugno 2011.

Dentone, P., Scritture femminili, intervista consultabile sul sito web della Casa Editrice

Scrittura \& Scritture: http://www.scritturascritture.it.

González de Sande, M., prefazione al testo: A. Assini, Las rosas de Cordoba, op.cit..

Guarinieri, P., intervista per «Leggere tutti», giugno 2011.

Pirrrota, L., recensione di Gilles, che amava Jeanne per «La Scrittura», autunno 1998.

----, recensione di Il fuoco e la creta. Storie comuni nella Napoli straordinaria del 1799 su «Il

Convivio», ottobre 2003.

----, prefazione al testo Il bacio del diavolo. Storia della contessa sanguinaria, op.cit.

Pizzarelli, L., Storia di una serial killer in "Un sorso di arsenico", presentazione del testo per «Foggia\&Foggia.com», 12 marzo 2010.

Sacconi, S., recensione di Nella foresta di Soignes su «Il Ponte», 2001.

Tavolino riservato a Adriana Assini, intervista realizzata da "icaffeculturali.com", http://www.icaffeculturali.com/comunita/tavolino/AdrianaAssini/Adriana\%20 Assini.htm.

Topa, P., presentazione di Le evangeliste di Bruges su «Il Convivio», novembre 2004.

Valentini, S., recensione di Le rose di Cordova per «La Nuova Tribuna Letteraria», luglio 2008.

\section{ELENCO DEI NOMI:}

Alighieri, Dante.

Assini, Adriana.

Aversa, Yvonne.

Báthory, Erzsébet.

Benedetto de, Cristoforo.

Bulgakov, Michail.

Caracciolo, Marina.

Carlo d'Asburgo.

Deleuze, Jean.

Dentone, Paola.

Ferdinando d'Aragona.

Filippo d'Asburgo.

Giovanna d'Arco.

Giovanna di Castiglia

Goes van der, Hugo.

Gonzáles de Sande, Mercedes.

Guarnieri, Paola.

Isabella di Castiglia.

Laval de, Gilles.

Mancinelli, Laura.

Moncada de, Hugo.

Mozart, Wolfgang Amadeus.

Pizan de, Christine.

Pirrotta, Luciano.

Pizzarelli, Lucia.

Rossini, Gioacchino.

Sacconi, Stefano.

Semiramide.

Squarcialupo, Gian Luca.

Tofana, Giulia.

Topa, Pacifico.

Valentini, Stefano.

Vassalli, Sebastiano.

Voltaire (François-Marie Arouet).

Revista Internacional de Culturas y Literaturas, octubre 201 
Adriana Assini. La gioia del colore

Vivaldi, Antonio. 